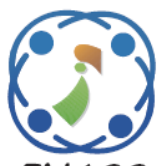

\title{
A Novel Building Detection Technique Using Image Descriptor CPMID in Urban Area
}

\author{
Kala Singarayan ${ }^{1 *}$ \\ Jeyakumar Muthayyan Kamalam² \\ ${ }^{1}$ Department of Computer Science, Bharathiar University, Coimbatore, India \\ ${ }^{2}$ Department of Computer Application, Noorul Islam Centre for Higher Education, Kumaracoil, India \\ * Corresponding author's Email: kalajk1980@gmail.com
}

\begin{abstract}
Building Detection in urban areas is a crucial and challenging part of satellite imagery but it suffers the issue of inaccuracy. This research proposes a novel texture descriptor for segmentation of buildings in urban areas. This texture descriptor is designed and derived from the practical assessment of Chess piece movements telescoped with King, Queen, Bishop, Knight, and Rook and henceforth named as Chess Piece Movement based Image Descriptor (CPMID). The proposed method is hence named as Building Detection using CPMID; FCM and Morphology operations (BD-CPMIDFM). The proposed descriptor is empowered with data acquisition in all permitted directions of the specified Chess pieces which provides the texture image descriptor with higher accuracy that can be used in building detection in urban areas. The building detection is achieved through Morphology Operations Induced Fuzzy C-Means (MOI-FCM) segmentation and this output is post-processed through morphological Erosion and Dilation operations. The proposed building detection method shows significant levels of improvement in accuracy than existing versions in literature. Experimental results show that the proposed method improves the average segmentation accuracy up to 91.514.
\end{abstract}

Keywords: Building detection, FCM, CPMID, Morphology operators, Satellite images.

\section{Introduction}

Urbanization requires the government agencies and architects to modernize land diagrams and develop long period plans consequently. Very high resolution satellite images offer precious data that cover up huge regions for urbanization purpose. But their manual assessment is very tough and prone to fault [1]. Satellite images provide the information for geography, cartography, surveillance, city planning and so on [2]. It is very difficult for a human expert to manually monitor buildings in satellite images [3]. The building detection methods of literature suffer from lack of building segmentation accuracy. Therefore, it is an urge to develop robust technique for detection of buildings from satellite images with significant improvement in accuracy. To address this issue, research community introduced automated building detection methods. S. Chen et. al [6] presents range detector for building detection, but it suffers false alarm such as some of the man-made objects like garages, basket and tennis courts are identified as buildings and also this method is not suitable for large buildings with irregular shape, width, etc. Q. Zhang et.al [7] proposes a framework using MBI method but it fails in many situations. That is many buildings are not detected, very high computational efficiency and also it is suitable in rural areas. Many objects like car, road, bare land and open spaces are detected as buildings. D. konstantinidis et al. [8] proposes a method has two modules. Feature detector and refinement process. But this method suffers some of the buildings are lost. Some buildings are partially or falsely extracted and also false positives are occurring. J. D. Wegner et al. [10] presented a method for building detection based on object features which are extracted from high resolution interferometry Synthetic Aperture Radar (SAR) data. This approach is limited by the regular 
grid structure of image patches. A. Urlapathy et al. [11] proposed a template matching algorithm and uses SVM classifier for differentiation of buildings from other structures. The issue behind this is that the edges of detected building structures are not smooth as the original ones. A. Ferro et al. [12] presented a method based on the extraction of a set of low-level features from the images and on their composition to more structured primitives using a production system. For small buildings, this technique shows worse detection and an increased number of false alarms. C. Tao et al. [13] proposed a method with two steps; i) extracts a large set of corners from input image by an improved Harris corner detector (ii) to identify the candidate regions. But for real application the false alarm rate is still high. L. Deng et al. [14] proposed a technique that integrates time-frequency decomposition and entropy/alpha-Wishart classifier of POLSAR images. This integration result reaches only around $80 \%$ of accuracy. H. C. Fan et al. [15] proposed an approach for identifying man-made objects from MLS (Mobile Laser Scanning). In this approach, Geo-referenced MLS raw data is taken as input and Pre-processing is done along with detecting seed points and identifying man-made objects. This approach detects only the man- made objects on both sides of the street. Limitations of this works are identifying trucks as small houses and classify some isolated short fences as cars. Wu et al. [16] proposed the nonzero-mean model based multi-variant normal distribution. This method considers the scattering vector of Polari-metric SAR (polSAR) images as input. Several objects like sparse trees and rocks may introduce errors in the segmented building result. N. Li et al. [17] proposed a texture measure method which can be implemented in different ways. But this technique is affected by the IFS (Intuitionistic Fuzzy Set) operators. It effectively identifies the build-up areas with considerable false alarms. W. Wu et al. [18] proposed the polSAR image feature extraction method which is developed by combining the Spherically Invariant Random Vector (SIRV) product model with the TimeFrequency (TF) analysis technology. The drawback of TF-texture based technique is that it is not sensitive to the differences of natural targets. J. Gu et al. [19] introduced a fuzzy double c-means clustering algorithm based on sparse selfrepresentation (FDCM-SSR). This algorithm clusters two data sets simultaneously i.e., the basic feature set and the discriminant feature set. This algorithm is applied in data clustering and image segmentation. F. Li et al. [20] provides a variant of FCM clustering algorithm called as Fuzzy Local
Information LP (FLIL $)$ clustering for image segmentation. This algorithm outperforms in terms of noise immunity and segmentation accuracy compared to other existing variants of FCM. But this algorithm suffers by computational complexity and time complexity. K. H. Memon et al. [21] introduced a variant of FCM called generalized Fuzzy C-Means clustering algorithm with local information (GFCMLI). In order to rectify the disadvantages of FCM, this algorithm added a new factor in the FCM objective function. This algorithm overcomes the drawback of FCM on poor performance when using clusters with different sizes and densities.

The existing algorithms in literature are suffered by the issue of inaccuracy like high false alarm, 'misidentification of trucks, car, rock and other man-made objects as buildings'. This research proposes a novel texture descriptor for the segmentation of buildings in urban areas. This texture descriptor is designed and derived from the practical assessment of Chess piece movements telescoped with King, Queen, Bishop, Knight and Rook and henceforth named as Chess Piece Movement based Image Descriptor (CPMID).The proposed method is hence named as Building Detection using CPMID, FCM and Morphology operations (BD-CPMIDFM). The proposed descriptor is guided by data acquisition in all permitted directions of the specified Chess pieces and hence a higher accuracy providing texture image descriptor is generated which can be efficiently used for detecting buildings in urban areas. The building detection process is reached through the Morphology Operations Induced Fuzzy $C$ Means (MOI-FCM) segmentation and this output is post-processed through morphological Erosion and Dilation operations. The proposed building detection method overcomes the drawback of state of art methods of literature due to the significant improvement in building segmentation accuracy.

The Section 2 provides the working methodology of proposed building detection method. Section 3 shows the Results and Discussions and Section 4 expresses the conclusion of this manuscript.

\section{Proposed method}

The Proposed method is constructed using the following three components.

- Image descriptor

- FCM [4]

- Morphology based Post processing [5] 


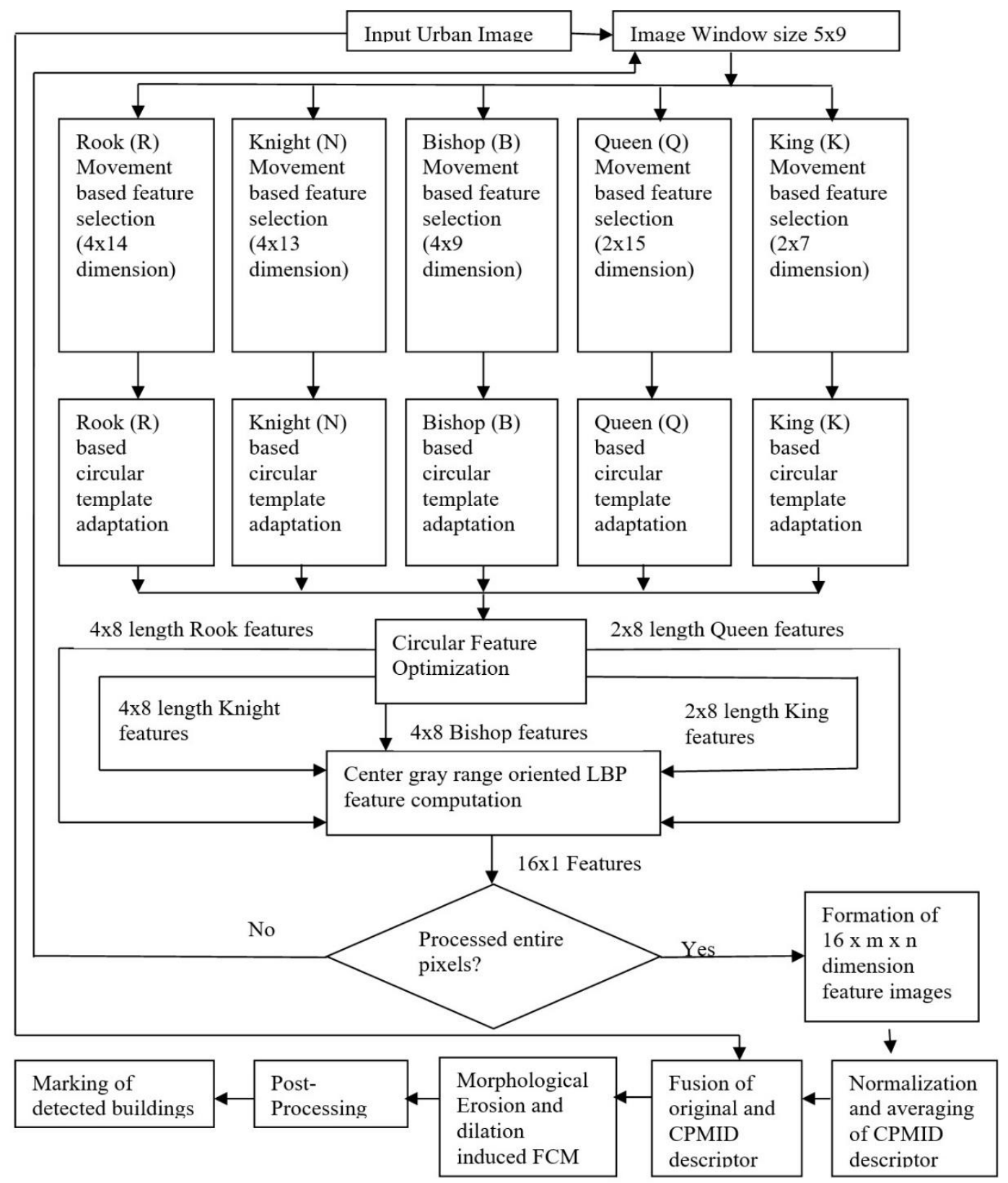

Figure. 1 Architecture diagram of the proposed building detection method

This manuscript proposes a novel method BDCPMIDFM for efficient building detection using three major concepts including an image feature descriptor, unsupervised clustering and postprocessing. The Gray scale satellite image is given as input to this system and the building area are detected and marked by the proposed method. A new image feature descriptor namely CPMID is proposed to extract the feature data from the input image. In this CPMID descriptor, the feature is extracted using the directions in which the Chess pieces move. The clustering process is achieved through a new variation of Fuzzy C-Means (FCM) method namely MOI-FCM. The design of MOIFCM is influenced by Morphological Erosion and
Dilation operations. The Morphological Erosion and Dilation operations induce the FCM to produce the efficient clustered result. Post-processing is attached to recover and refine the clustered building output. Post-processing is involved with the morphological operations. The following methods are the main modules of the proposed BD-CPMIDFM method.

- CPMID image descriptor

- Morphological erosion and dilation induced FCM (MOI-FCM).

- Refinement using morphological post processing.

The Fig.1 explains the overall architecture of the proposed method to have better understanding. 


\subsection{CPMID descriptor}

The CPMID image descriptor extracts spatial features (intensity features) based on the directions in which the Chess pieces move. The Chess pieces namely Rook, Knight, Bishop, Queen, and King are employed to extract features. The Pawn is not utilized here in to avoid the excess quantity of features because in the game of Chess there are sixteen pawns which are too much for feature computation. Fig.2 illustrates the name, shape, and locations of Chess pieces. In this research, totally sixteen feature images are derived from sixteen Chess pieces of Rook, Knight, Bishop, Queen, and King. Among these sixteen feature images four feature images are drawn from four Rooks, four feature images are drawn from four Knights, four feature images are drawn from four Bishops, two Queen pieces assists in generating two feature images and two King pieces aids to construct two feature images. The feature image detection process is carried out by a $5 \times 8$ size overlapped window. For experimental purpose, the sixteen Chess pieces are positioned based on Fig.3 which also demonstrates the $5 \times 8$ window.

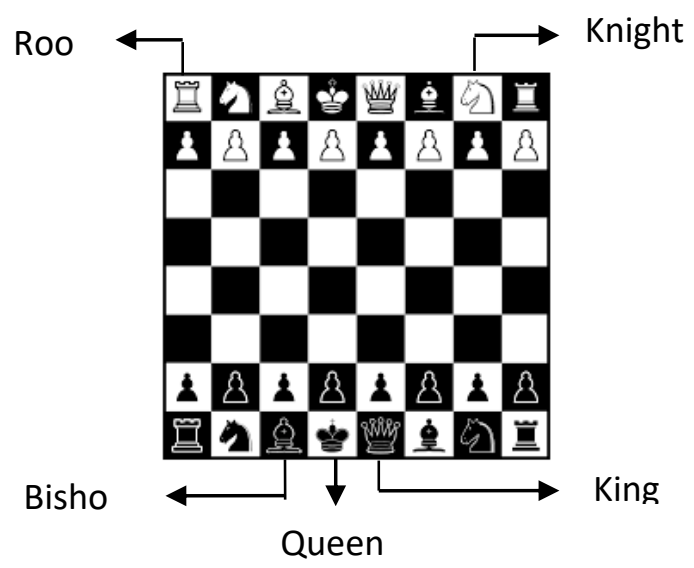

Figure.2 Representation of Chess pieces and their locations

\begin{tabular}{|l|l|l|l|l|l|l|l|}
\hline R0 & N0 & B0 & Q0 & K0 & B1 & N1 & R1 \\
\hline & & & & & & & \\
\hline & & & & i, $j$ & & & \\
\hline & & & & & & & \\
\hline R3 & N3 & B3 & Q1 & K1 & B2 & N2 & R2 \\
\hline
\end{tabular}

Figure. 3 Window structure and Chess piece positioning

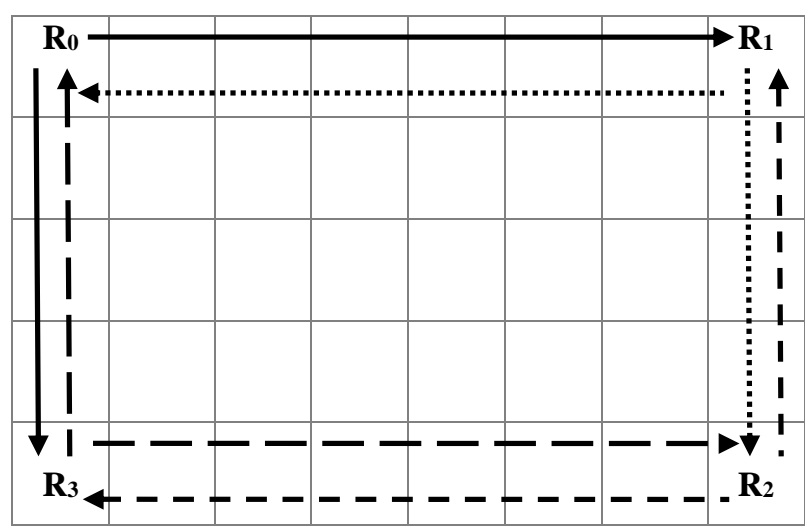

Figure. 4 Rook movement representation

\subsubsection{Rook movement based features}

In Chess, 4 Rooks are positioned at their specific squares. The positions and their possible movements are depicted in Fig.4. The Rook_0 based features are derived from the input image using the $5 \times 8$ window structure and the Rook_0 movement intensity feature $F_{R 0}$ is collected by Eq. (1).

$$
\begin{aligned}
& F_{R 0} \\
& =\left\{\begin{array}{c}
\mathrm{I}(\mathrm{i}-2, \mathrm{j}-4), \mathrm{I}(\mathrm{i}-1, \mathrm{j}-4), \mathrm{I}(\mathrm{i}+0, \mathrm{j}-4), \\
I(i+1, j-4), I(i+2, j-4), I(i-2, j-4), \\
I(i-2, j-3), I(i-2, j-2), I(i-2, j-1), \\
I(i-2, j+0), I(i-2, j+1), I(i-2, j+2), \\
I(i-2, j+3)
\end{array}\right\}
\end{aligned}
$$

The feature length of Rook_0 is derived as 14 because of 5 vertical move locations, 8 horizontal move locationsand a center pixel I $(i, j)$.

The Rook_1 feature $F_{R I}$ is derived using Eq. (2).

$$
\begin{aligned}
& F_{R 1} \\
& =\left\{\begin{array}{c}
I(i-2, j+3), I(i-1, j+3), I(i+0, j+3), \\
I(i+1, j+3), I(i+2, j+3), I(i-2, j+3), \\
I(i-2, j+2), I(i-2, j+1), I(i-2, j+0), \\
I(i-2, j-1), I(i-2, j-2), I(i-2, j-3), \\
I(i-2, j-4)
\end{array}\right\}
\end{aligned}
$$

The Rook_2 feature $F_{R 2}$ is computed using Eq. (3).

$$
\begin{aligned}
& F_{R 2}= \\
& \left\{\begin{array}{c}
I(i+2, j-4), I(i+1, j-4), I(i+0, j-4), \\
I(i-1, j-4), I(i-2, j-4), I(i+2, j-4), \\
I(i+2, j-3), I(i+2, j-2), I(i+2, j-1), \\
I(i+2, j+0), I(i+2, j+1), I(i+2, j+2), \\
I(i+2, j+3)
\end{array}\right\}
\end{aligned}
$$


The Rook_3 feature $F_{R 3}$ is computed using Eq. (4).

$$
\begin{aligned}
& F_{R 3} \\
& =\left\{\begin{array}{c}
I(i+2, j+3), I(i+1, j+3), I(i+0, j+3), \\
I(i-1, j+3), I(i-2, j+3), I(i+2, j+3), \\
I(i+2, j+2), I(i+2, j+1), I(i+2, j+0), \\
I(i+2, j-1), I(i+2, j-2), I(i+2, j-3), \\
I(i+2, j-4)
\end{array}\right\}
\end{aligned}
$$

The Rook based features are restructured based on the circular format. The circular template is shown in Fig. 5.

The Fig.5 is divided into four zones which are fixed for Rook_0, Rook_1, Rook_2, and Rook_3 features. The entire circular template constitutes of five layers. The Rook_0 features are filled up in the circular format which is guided by the Rook_0 zone. Similarly the other feature sets R1, R2 and R3 are converted into circular format based on their zone design. The element distribution in circular format for a single zone is defined based on the following two rules.

After filling, no circular layers will be partially filled. The specified Chess piece related feature should be distributed without any balance. Each zone is divided as bins namely $B I N_{S}, B I N_{T}, B I N_{U}$, $B I N V, B I N_{W}, B I N_{X}, B I N_{Y}, B I N_{Z}$. The Rook_0 zone bins are defined based on Eqs. (5) to (12).

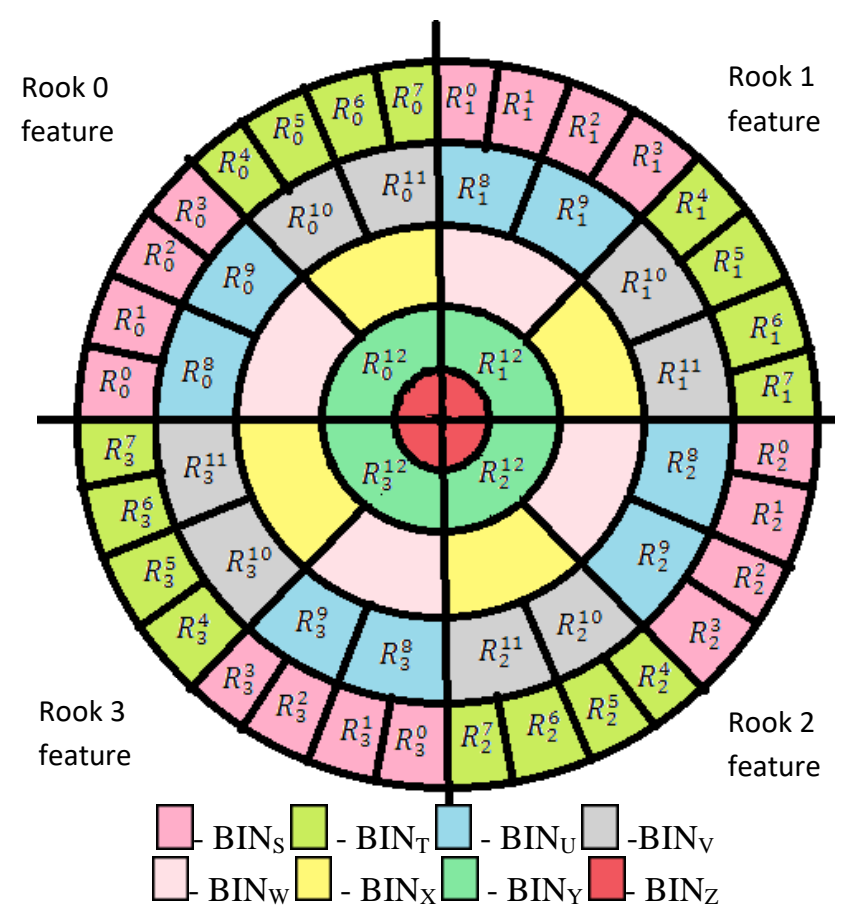

Figure. 5 Circular template for Rook movement

$$
\begin{aligned}
& B I N_{S}^{0}=\left\{R_{0}^{0}, R_{0}^{1}, R_{0}^{2}, R_{0}^{3}\right\} \\
& B I N_{T}^{0}=\left\{R_{0}^{4}, R_{0}^{5}, R_{0}^{6}, R_{0}^{7}\right\} \\
& B I N_{U}^{0}=\left\{R_{0}^{8}, R_{0}^{9}\right\} \\
& B I N_{V}^{0}=\left\{R_{0}^{10}, R_{0}^{11}\right\} \\
& B I N_{W}^{0}=\{0\} \\
& B I N_{X}^{0}=\{0\} \\
& B I N_{Y}^{0}=\left\{R_{0}^{12}\right\} \\
& B I N_{Z}^{0}=\{\mathrm{I}(\mathrm{i}, \mathrm{j})\}
\end{aligned}
$$

The BIN ${ }_{W}$ is fixed with 0 because in Fig.5 it is an empty bin. Similarly BIN $\mathrm{B}_{\mathrm{X}}$ is also fixed with 0 . The new 8 components based on bins are computed using Eqs. (13) to (20). These eight components are named as a, b, c, d, e, f, g and h.

$$
\begin{aligned}
& \mathrm{a}=\operatorname{AVG}\left(B I N_{S}^{0}\right) \\
& \mathrm{b}=\operatorname{AVG}\left(B I N_{T}^{0}\right) \\
& \mathrm{c}=\operatorname{AVG}\left(B I N_{U}^{0}\right) \\
& \mathrm{d}=\operatorname{AVG}\left(B I N_{V}^{0}\right) \\
& \mathrm{e}=0 \\
& \mathrm{f}=0 \\
& \mathrm{~g}=\mathrm{BIN}_{\mathrm{Y}}^{0} \\
& \mathrm{~h}=\mathrm{BIN}_{\mathrm{Z}}^{0}
\end{aligned}
$$

The single gray value LBP can be computed using Eqs. (21) to (28).

$$
\begin{aligned}
L B P & =0 \\
L B P & =\left\{\begin{array}{ll}
\mathrm{LBP}+128, \text { if }((\mathrm{I}(\mathrm{i}, \mathrm{j})-\mathrm{a}) \geq 127) \\
\mathrm{LBP} & \text { else }
\end{array}\right\} \\
L B P & =\left\{\begin{array}{ll}
\mathrm{LBP}+64, \text { if }((\mathrm{I}(\mathrm{i}, \mathrm{j})-\mathrm{b}) \geq 127) \\
\mathrm{LBP} & \text { else }
\end{array}\right\} \\
L B P & =\left\{\begin{array}{cc}
\mathrm{LBP}+32, \text { if }((\mathrm{I}(\mathrm{i}, \mathrm{j})-\mathrm{c}) \geq 127) \\
\mathrm{LBP} \quad \text { else }
\end{array}\right\}
\end{aligned}
$$




\begin{tabular}{|l|l|l|}
\hline$a$ & $b$ & $C$ \\
\hline$h$ & I $(i, j)$ & $D$ \\
\hline$g$ & $f$ & $E$ \\
\hline
\end{tabular}

Figure. 6 LBP element's position representation.

$$
\begin{aligned}
L B P & =\left\{\begin{array}{ll}
\mathrm{LBP}+16, \text { if }((\mathrm{I}(\mathrm{i}, \mathrm{j})-\mathrm{d}) \geq 127) \\
\mathrm{LBP} \quad \text { else }
\end{array}\right\} \\
L B P & =\left\{\begin{array}{ll}
\mathrm{LBP}+8, \text { if }((\mathrm{I}(\mathrm{i}, \mathrm{j})-\mathrm{e}) \geq 127) \\
\mathrm{LBP} \quad \text { else }
\end{array}\right\} \\
L B P & =\left\{\begin{array}{ll}
\mathrm{LBP}+4, \text { if }((\mathrm{I}(\mathrm{i}, \mathrm{j})-\mathrm{f}) \geq 127) \\
\mathrm{LBP} \text { else }
\end{array}\right\} \\
L B P & =\left\{\begin{array}{ll}
\mathrm{LBP}+2, \text { if }((\mathrm{I}(\mathrm{i}, \mathrm{j})-\mathrm{g}) \geq 127) \\
\mathrm{LBP} \quad \text { else }
\end{array}\right\} \\
L B P & =\left\{\begin{array}{ll}
\mathrm{LBP}+1, \text { if }((\mathrm{I}(\mathrm{i}, \mathrm{j})-\mathrm{h}) \geq 127) \\
\mathrm{LBP} \quad \text { else }
\end{array}\right\}
\end{aligned}
$$

The feature image $I R_{0}$ of Rook_0 is obtained via LBP using Eq. (29). The terms $H$ and $W$ are the original height and width of image.

$$
\begin{gathered}
I R_{0}^{i, j}=L B P \\
\mathrm{i} \in[0, \mathrm{H}-1] \quad \mathrm{j} \in[0, \mathrm{~W}-1]
\end{gathered}
$$

Similar to $I R_{0}$ computation, image features $I R_{1}, I R_{2}$ and $I R_{3}$ are also obtained.

\subsubsection{Knight movement based features}

In Chess, four knights are positioned at their specific squares. The positions and their possible movements are depicted in Fig.7.

Fig. 7 Knights movement representation. The Knight_0 based features are derived from the input image using $5 \times 8$ window structure and the Knight_0 movement intensity feature $F_{N O}$ is collected by Eq. (30).

$$
\begin{aligned}
& F_{N 0} \\
& =\left\{\begin{array}{l}
\mathrm{I}(\mathrm{i}-2, \mathrm{j}-3), \mathrm{I}(\mathrm{i}-1, \mathrm{j}-3), \mathrm{I}(\mathrm{i}+0, \mathrm{j}-3), \\
\mathrm{I}(\mathrm{i}+0, \mathrm{j}-4), \mathrm{I}(\mathrm{i}-2, \mathrm{j}-3), \mathrm{I}(\mathrm{i}-1, \mathrm{j}-3), \\
\mathrm{I}(\mathrm{i}+0, \mathrm{j}-3), \mathrm{I}(\mathrm{i}+0, \mathrm{j}-2), \mathrm{I}(\mathrm{i}-2, \mathrm{j}-3), \\
\mathrm{I}(\mathrm{i}-2, \mathrm{j}-2), \mathrm{I}(\mathrm{i}-2, \mathrm{j}-1), \mathrm{I}(\mathrm{i}-1, \mathrm{j}-1)
\end{array}\right\}
\end{aligned}
$$

The Knight_1 feature $F_{N l}$ is obtained by using Eq. (31).

$$
\begin{aligned}
& F_{N 1}(\mathrm{i}-2, \mathrm{j}+2), I(\mathrm{i}-1, \mathrm{j}+2), \mathrm{I}(\mathrm{i}+0, \mathrm{j}+2), \\
& =\left\{\begin{array}{l}
\mathrm{I}(\mathrm{i}+0, \mathrm{j}+3), \mathrm{I}(\mathrm{i}-2, \mathrm{j}+2), \mathrm{I}(\mathrm{i}-1, \mathrm{j}+2), \\
\mathrm{I}(\mathrm{i}+0, \mathrm{j}+2), \mathrm{I}(\mathrm{i}+0, \mathrm{j}+1), \mathrm{I}(\mathrm{i}-2, \mathrm{j}+2), \\
\mathrm{I}(\mathrm{i}-2, \mathrm{j}+1), \mathrm{I}(\mathrm{i}-2, \mathrm{j}+0), \mathrm{I}(\mathrm{i}-1, \mathrm{j}+0)
\end{array}\right\}
\end{aligned}
$$

The Knight_2 feature $F_{N 2}$ is obtained by using Eq. (32).

$F_{N 2}(\mathrm{I}+2, j-3), I(i+1, j-3), I(i+0, j-3)$,
$=\left\{\begin{array}{c}I(i+0, j-4), I(i+2, j-3), I(i+1, j-3), \\ I(i+0, j-3), I(i+0, j-2), I(i+2, j-3), \\ I(i+2, j-2), I(i+2, j-1), I(i+1, j-1)\end{array}\right\}$

The Knight_3 feature $F_{N 3}$ is obtained using Eq. (33).

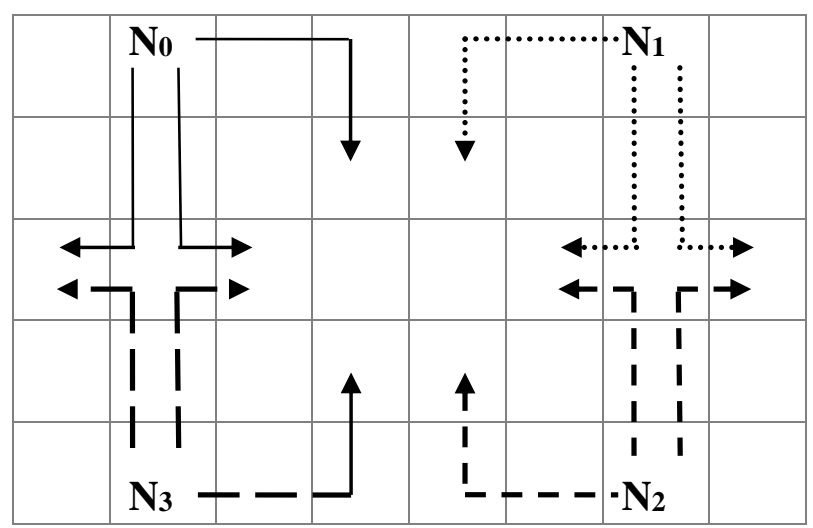

Figure. 7 Knights movement representation

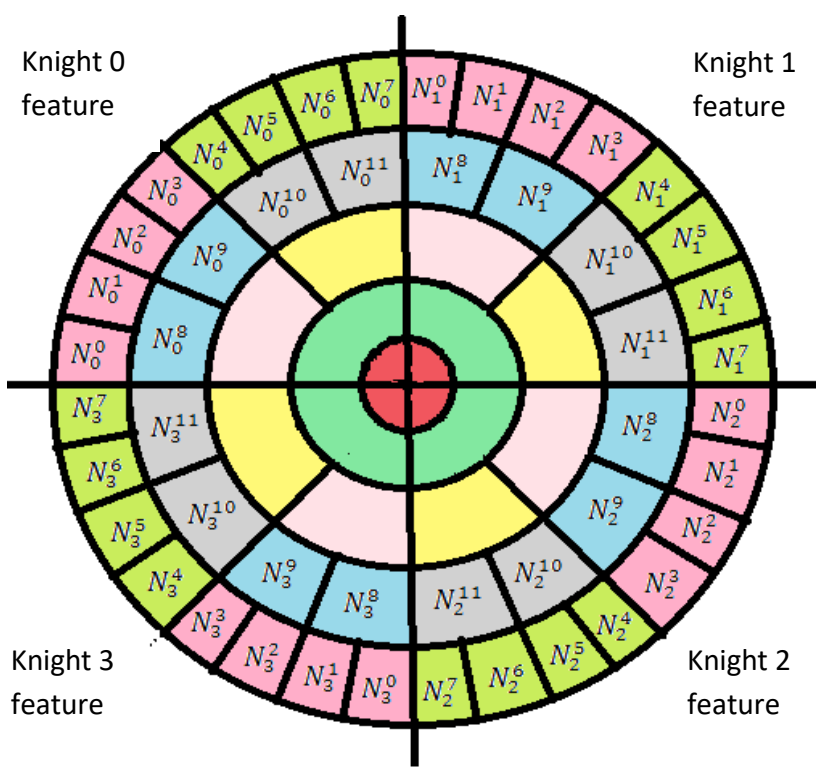

Figure. 8 Circular template for Knight Movement 


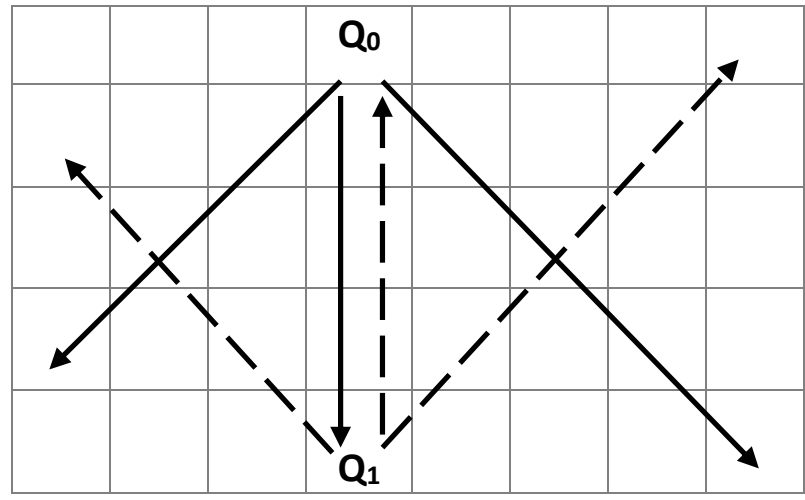

Figure. 9 Bishop movements representation

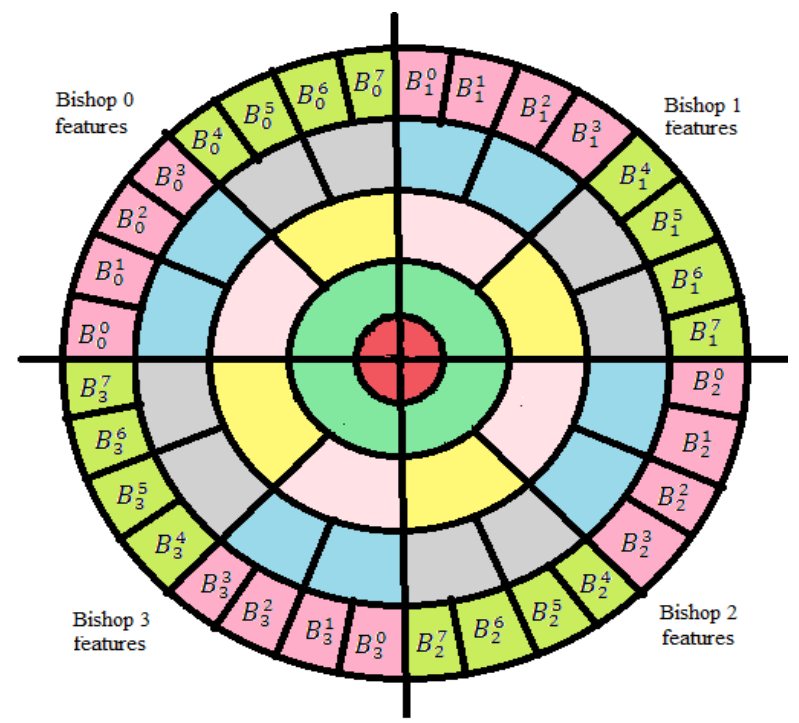

Figure. 10 Circular template for Bishop movements

$$
\begin{aligned}
& F_{N 3} \\
& =\left\{\begin{array}{l}
I(i+2, j+2), I(i+1, j+2), I(i+0, j+2), \\
I(i+0, j+3), I(i+2, j+2), I(i+1, j+2), \\
I(i+0, j+2), I(i+0, j+1), I(i+2, j+2), \\
I(i+2, j+1), I(i+2, j+0), I(i+1, j+0)
\end{array}\right\}
\end{aligned}
$$

The Knight based features are restructured based on the circular format which is shown in Fig.8.

The Knight_0 based feature count is 12. The BIN separation and 8 component extraction are performed as similar with Rook_0 piece procedure. The feature image $I N_{0}$ of Knight_0 is obtained using LBP computation using Eqs. (21) to (28). The image features $I N_{l}, I N_{2}$ and $I N_{3}$ are obtained in similar with $I N_{0}$ computation.

\subsubsection{Bishop movement based features}

In Chess, four Bishops are positioned at their specific squares. The positions and their possible movements are depicted in Fig.9.

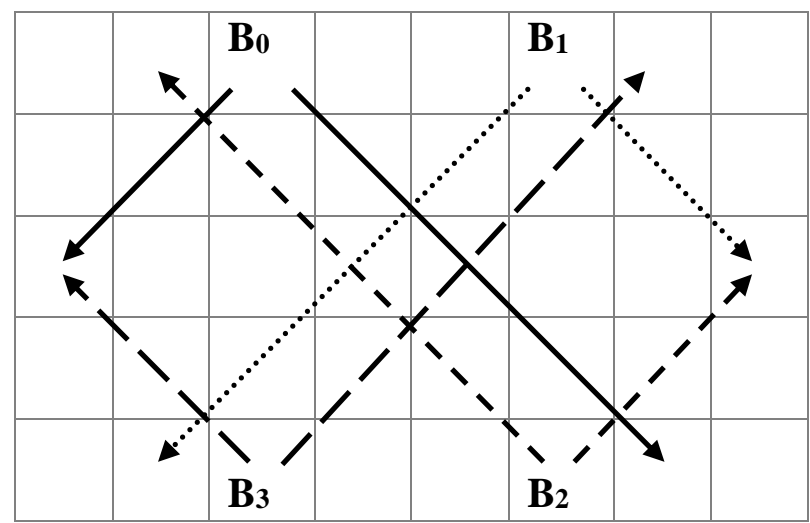

Figure. 11 Queen based movement representation

The Bishop_0 based features are derived from the input image using $5 \times 8$ window structure and the Bishop_0 movement intensity feature $F_{B O}$ is collected by Eq. (34).

$$
F_{B 0}=\left\{\begin{array}{c}
I(i-2, j-2), I(i-1, j-3), I(i+0, j-4), \\
I(i+1, j-3), I(i+2, j-2), I(i-2, j-2), \\
I(i-1, j-1), I(i+0, j+0), I(i+1, j+1), \\
I(i+2, j+2)
\end{array}\right\}
$$

The Bishop_1 feature $F_{B I}$ is obtained by using Eq. (35).

$$
\begin{aligned}
& F_{B 1}(\mathrm{I}(\mathrm{i}-2, \mathrm{j}+1), \mathrm{I}(\mathrm{i}-1, \mathrm{j}+2), \mathrm{I}(\mathrm{i}+0, \mathrm{j}+3), \\
& =\left\{\begin{array}{c}
\mathrm{I}(\mathrm{i}+1, \mathrm{j}+2), \mathrm{I}(\mathrm{i}+2, \mathrm{j}+1), \mathrm{I}(\mathrm{i}-2, \mathrm{j}+1), \\
\mathrm{I}(\mathrm{i}-1, \mathrm{j}+0), \mathrm{I}(\mathrm{i}+0, \mathrm{j}-1), \mathrm{I}(\mathrm{i}+1, \mathrm{j}-2), \\
\mathrm{I}(\mathrm{i}+2, \mathrm{j}+3)
\end{array}\right\}
\end{aligned}
$$

The Bishop_2 feature $F_{B 2}$ is obtained by using Eq. (36).

$$
\begin{aligned}
& F_{B 2} \\
& =\left\{\begin{array}{c}
I(i+2, j-2), I(i+1, j-3), I(i+0, j-4), \\
I(i-1, j-3), I(i-2, j-2), I(i+2, j-2), \\
I(i+1, j-1), I(i+0, j+0), I(i-1, j+1), \\
I(i-2, j+2)
\end{array}\right\}
\end{aligned}
$$

The Bishop_3 feature $F_{B 3}$ is obtained by using Eq. (37).

$$
\begin{aligned}
& F_{B 3} \\
& =\left\{\begin{array}{c}
I(i+2, j+1), I(i+1, j+2), I(i+0, j+3), \\
I(i-1, j+2), I(i-2, j+1), I(i+2, j+1), \\
I(i+1, j+0), I(i+0, j-1), I(i-1, j-2), \\
I(i-2, j-3)
\end{array}\right\}
\end{aligned}
$$


The circular template of Bishop Movement is shown in Fig.10.

The Bishop_0 based feature count is 8 because of five long diagonal movements and three short movements. The BIN separation and 8 component extraction for Bishop_0 are performed. LBP computation is also performed to construct the Bishop_0 feature image $I B_{0}$. The image features $I B_{l}$, $I B_{2}$ and $I B_{3}$ are obtained similar to $I B_{0}$ computation.

\subsubsection{Queen movement based features}

In Chess, two Queens are positioned at their specific squares. The positions and their possible movements are depicted in Fig.11

The Queen_0 based feature is derived from the input image using $5 \times 8$ window structure and the Queen_0 movement intensity feature $F_{Q 0}$ is obtained by Eq. (38).

$$
\begin{aligned}
& F_{Q 0} \\
& =\left\{\begin{array}{c}
I(i-2, j-1), I(i-1, j-1), I(i+0, j-1), \\
I(i+1, j-1), I(i+2, j-1), I(i-2, j-1), \\
I(i-1, j-2), I(i+0, j-3), I(i+1, j-1), \\
I(i-2, j-1), I(i-1, j+0), I(i+0, j+1), \\
I(i+1, j+2), I(i+2, j+3)
\end{array}\right\}
\end{aligned}
$$

The Queen_1 feature $F_{Q l}$ is obtained using Eq. (39).

$$
\begin{aligned}
& F_{Q 1} \\
& =\left\{\begin{array}{l}
I(i+2, j-1), I(i+1, j-1), I(i+0, j-1), \\
I(i-1, j-1), I(i-2, j-1), I(i+2, j-1), \\
I(i+1, j-2), I(i+0, j-3), I(i+1, j-4), \\
I(i+2, j-1), I(i+1, j+0), I(i+1, j+1), \\
I(i-1, j+2), I(i-2, j+3)
\end{array}\right\}
\end{aligned}
$$

The Queen based features are restructured based on the circular format. The circular template is shown in Fig. 12.

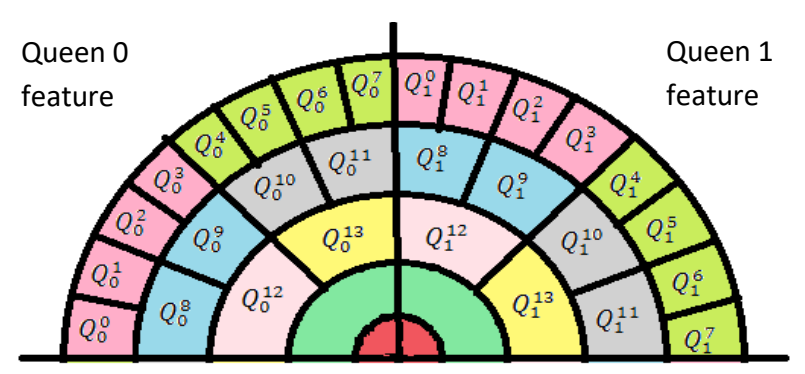

Figure. 12 Circular template for Queen movement

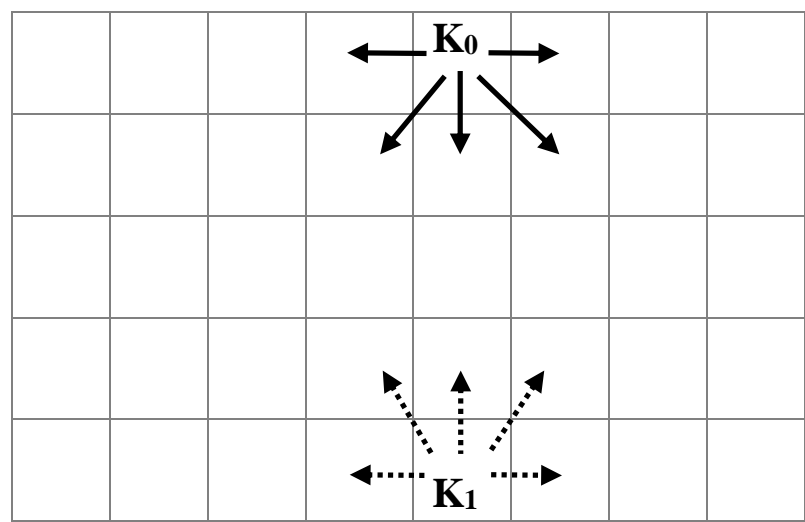

Figure. 13 King based movement representation

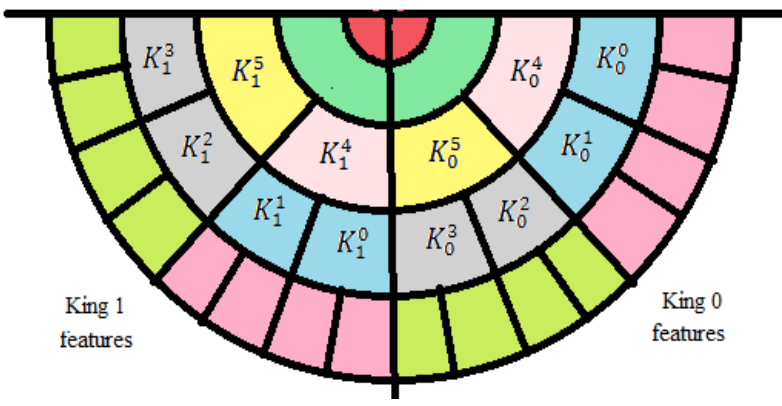

Figure. 14 King based movement representation

The Queen_0 based feature count is 14 because of short diagonal features, 5 vertical features and 5 long diagonal features. The BIN separation and eight component extraction of Queen_0 are performed. The LBP computation is again performed to generate Queen_0 feature image $I Q_{0}$. $I Q_{I}$ is also obtained similar to $I Q_{0}$ computation.

\subsubsection{King movement based features}

In Chess, two Kings are positioned at their specific squares. The positions and their possible movements are depicted in Fig.13. The King_0 based feature is derived from the input image using $5 \times 8$ window structure and the King_0 movement intensity feature $F_{K O}$ by Eq. (40).

$F_{K 0}=\left\{\begin{array}{c}I(i-2, j+0), \mathrm{I}(\mathrm{i}-2, \mathrm{j}+1), \mathrm{I}(\mathrm{i}-1, \mathrm{j}+1), \\ \mathrm{I}(\mathrm{i}-1, \mathrm{j}+0), \mathrm{I}(\mathrm{i}-1, \mathrm{j}-1), \mathrm{I}(\mathrm{i}-2, \mathrm{j}-1)\end{array}\right\}$

The King_1 feature $\mathrm{F}_{\mathrm{K} 1}$ is obtained using Eq. (41).

$F_{K 1}=$
$\left\{\begin{array}{c}I(i+2, j+0), I(i+2, j+1), I(i+1, j+1), \\ I(i+1, j+0), I(i+1, j-1), I(i+2, j-1)\end{array}\right\}$

The King based features are restructured based on the circular format. The circular template is shown in Fig.14. 
King_0 based feature count is 6 because of 5 movements and 1 piece location. The BIN separation and 8 component extraction for King_0 is performed. In order to construct the King_0 feature image $I K_{0}$ and King_1 feature image $I K_{l}$, the LBP computation is performed.

\subsubsection{Fusion process}

The Chess piece based movements provide 16 feature images and they can be represented by Eq. (42).

$$
F I_{i, j}=\left\{\begin{array}{c}
I R_{0}, I R_{1}, I R_{2}, I R_{3}, I N_{0}, I N_{1}, I N_{2} \\
I N_{3}, I B_{0}, I B_{1}, I B_{2}, I B_{3}, I Q_{0}, I Q_{1}, I K_{0}, I K_{1}
\end{array}\right\}
$$

A single feature image is derived from the featureimage- set $F I$ which includes the entire 16 feature images. The derived single feature image is known as CPMID descriptor. This single image formation is done through the fusion process which is influenced by mathematical averaging statistical function. The fusion process is described based on Eq. (43).

$$
C C M I D^{I, J}=\frac{1}{t} \sum_{k=0}^{t-1} \sum_{i=0}^{H-1} \sum_{j=0}^{W-1} F I_{i j}^{k}
$$

Where

$\mathrm{t}$ - Total no of image features (set as 16)

The CPMID descriptor contains feature information of input image.

\subsection{Morphological operations induced FCM}

Image segmentation algorithms are used to group the image data into meaningful information. The CPMID descriptor is used to segment the input image for detecting the building objects. Few algorithms are already released based on the variations in Fuzzy C-Means algorithm (FCM) such as Fuzzy Double C- Means based on sparse selfrepresentation (FDCM_SSR) [19], Fuzzy local information LP (FLILP) clustering [20], Generalized FCM clustering algorithm with local information (GFCMLI) [21] and Adaptive fuzzy local information C-means (ADFLICM) [22].

This paper introduces a novel image segmentation method namely Morphological Operations Induced FCM (MOI-FCM) which is the modified version of Fuzzy C-Means algorithm. This new algorithm is constructed by Fuzzy and Morphological operations. The Morphological operation of this paper is influenced by Erosion and Dilation processes. Fuzzy C-Means is a clustering method that was first developed by Dunn [23] in 1973 and Bezdek improved the algorithm in 1981[24].The Fuzzy logic allots each element of data belonging to a cluster with a degree of membership between the interval $[0,1]$.The traditional FCM algorithm is constructed by the following six steps.

1. Initialization.

2. Cluster center computation.

3. Distance measure calculation.

4. Updating Fuzzy membership matrix.

5. Objective function.

6. Defuzzification.

The Initialization process defines the number of clusters $C$, randomly selecting the position of the centers $\left(c_{j}\right)$ for each cluster and exponent value $(m)$. The cluster center computation calculates the new cluster center $\left(c_{j}\right)$ of the specified iteration. The distance measurement process measures the distance between the cluster head and data. The Fuzzymembership-updation process alters the membership matrix suitable to current iteration. The Objective function decides when to stop the iteration. The Defuzzification process groups the elements meaningfully from the recent membership matrix which yields the segmented image.

The proposed MOI-FCM method is the modified version of the FCM. The modifications are done in initialization process, cluster center computation and fuzzy membership matrix updation. These modifications are induced into the proposed MOIFCM method to enhance the segmentation accuracy. The MOI-FCM method invokes based on the morphological operations.

The Initialization process of MOI-FCM method assigns the number of clusters as 4 because major urban area images can be classified as 4 categories in which building class must be presented to reach the goal of this research paper. The exponent value $m$ is tuned as 2 because majority of FCM variations used this value to maintain the trade-off between real segmentation and over segmentation. This type of $m$ assignment is known as Fuzzy-II type segmentation which reduces the computational cost. The max-iteration is tuned with the value 50 which is enough to make excellent segmentation with less computational cost. In order to capture the enhanced segmentation output gradually, the min-imp (minimum improvement) is assigned as $1 \mathrm{e}-5$. The dimension of data is defined at the initialization stage as $[I H \times I W, 2]$ where $I H$ means image height, $I W$ means image width and numerical term 2 indicates the input vector count. The MOI-FCM 
method requires two image samples as input vectors. The first sample is provided by the CPMID image descriptor which has strong representation of building objects. The second sample is represented by the original input image which has intensity valued building objects. These two types of image representation provide a better segmentation to reach building detection effectively. This initial random cluster head $c_{j}$ generation and initial membership matrix $U$ generation are done only once at initialization stage. The dimension of membership matrix $U$ is $(I H \times I W, C)$.

The cluster head selection process chooses new cluster heads $c j$ related with each iteration using Eq. (44).

$$
C_{j}=\frac{\sum_{i=0}^{n-1}\left(U_{i j}\right)^{m} X i}{\sum_{i=0}^{n-1}\left(U_{i j}\right)^{m}}, \mathrm{j} \in[1, \mathrm{C}]
$$

The term $n$ means Total elements in each-sample vector (Here $n=I H \times I W$ ).

The proposed method makes a few meaningful modifications in the sample vectors to reduce the computational cost and to increase the robustness against noise. The input sample vectors are undergone morphological operations to remove the noises occurred at a periodic interval. The periodic interval here mentioned is 4 because the trial-anderror method applied on this implementation found that at each of the four successive intervals the noise may occur. Hence the interval- constant 4 is derived based on the results of simulation. The morphological operations such as Erosion and Dilation are used to remove the noise and to strengthen the building class for better segmentation. Morphological operators are used to make logical transformations based on comparison of pixel neighborhoods with a pattern [25]. This pattern is known as structuring element. The Dilation operation changes a foreground pixel to background if it has a background pixel as a 4 neighbor. The Erosion process shrinks the background. The Dilation process expands the foreground by changing background pixel to foreground if it has a foreground pixel as a 4 neighbor. The combination of erosion and dilation process can be used to remove the holes, noises and artifacts in the foreground. It can also be helpful to smooth region boundaries for shape analysis [26] [27]. The sample vector 1 (CPMID descriptor) is altered based on erosion and dilation using Eqs. (45) and (46).

$$
X_{i}^{j=1}=X_{i}^{j=1} \ominus S, \text { ifmod }(i t t, 4)=0 \& i t t \geq 4
$$

$$
\begin{gathered}
i \in[0, n-1] \\
X_{i}^{j=1}=X_{i}^{j=1} \oplus S, \text { if } \bmod (i t t, 4)=0 \& i t t \geq 4 \\
i \in[0, n-1]
\end{gathered}
$$

Where

$$
\begin{aligned}
& X_{i}^{j=1} \text { - CPMID descriptor which is stored in } \\
& \text { Sample vector } 1 \text {. } \\
& \ominus \text { - Erosion operator } \\
& \bigoplus \text { - Dilation operator } \\
& \mathrm{S} \text { - } 3 \times 3 \text { structuring element } \\
& \mathrm{n} \quad \text { - Length of vector } \\
& \text { itt } \quad \text { - fuzzy c-mean iteration }
\end{aligned}
$$

The sample vector 2 (spatial data) is modified based on erosion and dilation using Eqs. (47) and (48).

$$
X_{i}^{j=2}=X_{i}^{j=2} \ominus S, \text { ifmod }(i t t, 4)=0 \& i t t \geq 4
$$

$X_{i}^{j=2}=X_{i}^{j=2} \oplus S$, if $\bmod (i t t, 4)=0$ \& itt $\geq 4$

The $X_{i}^{j=2}$ means CPMID descriptor which is stored in sample vector 2 .

The input vectors of MOI-FCM are altered by a periodical interval to set new membership values to speed up the convergence state of objective function due to smoothness effect of erosion and dilation operations. This periodical interval is set to 4 and it should not be performed at the initial iteration. This process also yields robustness to noise. The novel contribution which is used to alter the sample vector is the key point for the success of MOI-FCM algorithm.

The noise and very small objects collapse the main object's fine segmentation by disturbing the foreground body of buildings in the satellite image and due to that the segmentation accuracy is reduced or obtained with minute grains. To make the segmentation noise tolerant, another contribution based on median filter is employed at fuzzy membership matrix without altering its structure. In the membership function, usually the magnitude of membership values is kept between 0 and 1 . The fuzzy membership function is temporarily defuzzified to get the temporary clustered image ITEMP by finding the index of maximum fuzzy membership- value provider. This $I_{T E M P}$ image is filled by values between 0 and 3 because the number of cluster is settled as 4 in this paper. A $3 \times 3$ size 
overlapped window $W_{3 \times 3}$ is selected from the corresponding pixel of the $i^{\text {th }}$ position element (in linear form) of cluster image $I_{T E M P}^{i, j}$. The unique information on the $W_{3 \times 3}$ Window is considered to find the unique information count $u$. If the unique count $u$ is equal to 2 or 3 then there is a chance of pixel values crossing the center index of the linear version of $W_{3 \times 3}$ which leads to the possibility of median calculation. The $\mathrm{W}_{3 \times 3}$ window values are converted into vector format $V_{k}$ where $\mathrm{k} \in[0,3 \times 3-1]$ from matrix format. This vector is sorted in ascending manner using Eq. (49).

$$
V_{\text {SORT }}=f_{\text {Sort }}\left(V_{k}\right)
$$

Where

$\mathrm{V}_{\text {SORT- }}$ sorted vector in ascending order

The center value of the $\mathrm{V}_{\text {SORT }}$ is traced out and stored as median value using Eq. (50).

$$
M E D=V_{S O R T}^{8 / 2}
$$

Where

$$
\text { MED - Median value of } \mathrm{V}_{\text {SORT }}
$$

The maximum membership value provider index means the better association with cluster head $c_{j}$ because of higher similarity of $i^{\text {th }}$ data with $C_{j}$ cluster head. The maximum membership value provider index is searched and stored in $I_{M A X}$ (referred as index of maximum). The magnitude of MED (Here it refers an index) and magnitude of $I_{M A X}$ are swapped to reduce the noise and minute objects if the unique count $u$ is between 2 and 3 by using Eq. (51).

Where

$$
\left.\begin{array}{c}
t=U_{i}^{j=M E D} \\
U_{i}^{M E D}=U_{i}^{I_{M A X}} \\
U_{i}^{I_{M A X}}=t
\end{array}\right\}
$$

\section{$\mathrm{t}$-Temporary variable used for swapping.}

The median value generally indicates the majority value of the neighbor data. The maximum membership value $U_{i}^{I_{M A X}}$ indicates the noisy data.

The objective function is computed based on the paper [28] and the objective function eliminates the iterative process when it reaches the minimum improvement min-imp. The Defuzzification process [28] is applied to extract the segmentation output. Normally the building intensities are set into high range and this important point is initialized to extract the building area with some disturbances. The high intensity providing segmentation index is separated as building images. The small size objects are appeared as noises in the segmented image and these small objects can be removed using a threshold range.

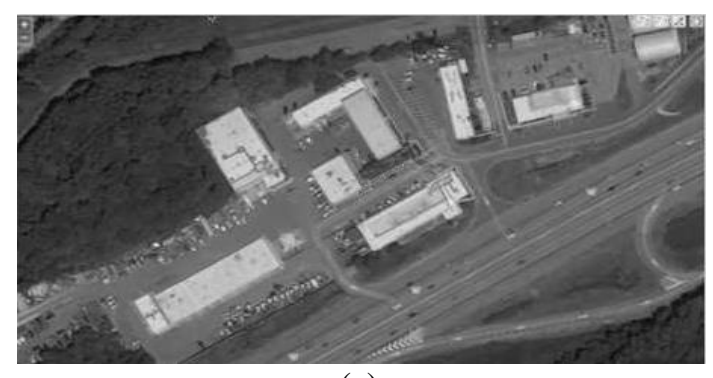

(a)

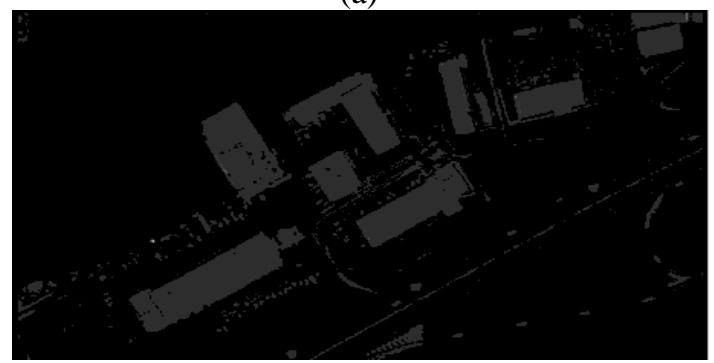

(b)

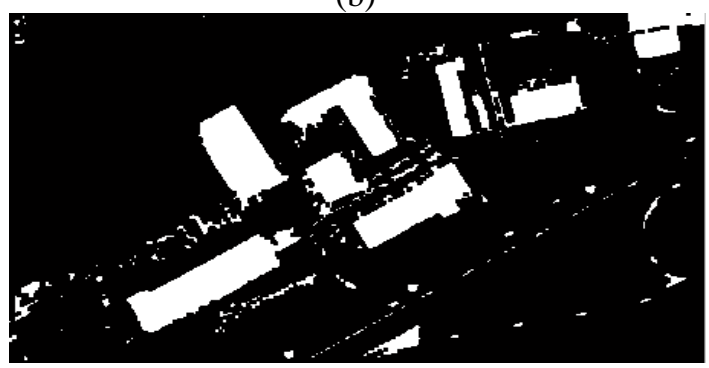

(c)

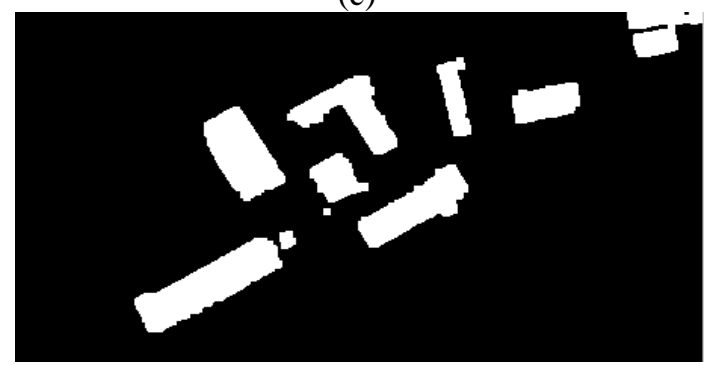

(d)

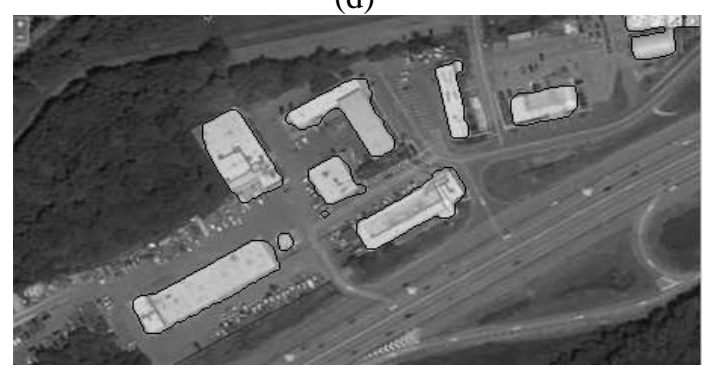

(e)

Figure. 15 Building detection using proposed method: (a) Input building image Building1, (b) Proposed CPMID descriptor result, (c) MOI-FCM based building detection, (d) Post processing result, and (e) Final building detection 


\subsection{Post processing}

The segmented buildings from MOI-FCM are affected by some noise information and holes. These disturbances are overcome by the refinement or post processing through Morphological operations. The MOI-FCM output is given as input to the post processing to erode the binary building image. The erosion process involves a square type structuring element with $3 \times 3$ window size. Finally, dilation process is performed with square structuring element with dimension of $3 \times 3$. This dilated output is the final building detection output in binary format.

\section{Analysis and discussion}

The proposed BD_CPMIDFM method makes an analysis with the following literature papers.

- Building Detection based on Range Detector (BD-RD) [6]

- Building Detection based on Morphological operations (BD-MO) [7]

- Building Detection based on HOG-LBP Features (BD-HL) [8]

Analysis is performed using the Advanced Space-borne Thermal Emission and Reflection Radiometer Global Emissivity Dataset (ASTERGED) [9].

The Fig. 15 depicts the building detection output of the proposed method. The Fig.15 (b) describes the CPMID output and Fig.15 (c) depicts the MOIFCM output on building segmentation. The Fig.15 (d) shows the post processing result and Fig.15 (e) describes the final building detection output. In Fig.15 (e) the marked buildings are projected on the original image.

The Fig.16 depicts the sample screens of the proposed method on building segmentation using Building2 image.

MSE computation is done by using Eq. (52).

$$
M S E=\frac{1}{P x Q} \quad \sum_{i=1}^{p} \sum_{j=1}^{q}\left(I(i, j)-I^{\prime}(i, j)\right)^{2}
$$

Where

$$
\begin{array}{lll}
\text { P } & - & \text { Height of the image } \\
\mathrm{Q} & - & \text { Width of the image } \\
\mathrm{I} & - & \text { Segmented Image } \\
\mathrm{I} & - & \text { Ground-truth Image }
\end{array}
$$

MSE analysis points out the Mean Square Error on building segmentation. The MSE computation is performed by using the two parameters BuildingSegmented-Output and Ground-truth-image. The MSE computation is done using Eq. (52). Lesser score in MSE provides best segmentation method and vice-versa. Table 1 show that the proposed method possesses least score in MSE while other methods are not. The least MSE value provided by the proposed method is 0.009 for the Building4 image and 0.050 for the second best segmentation

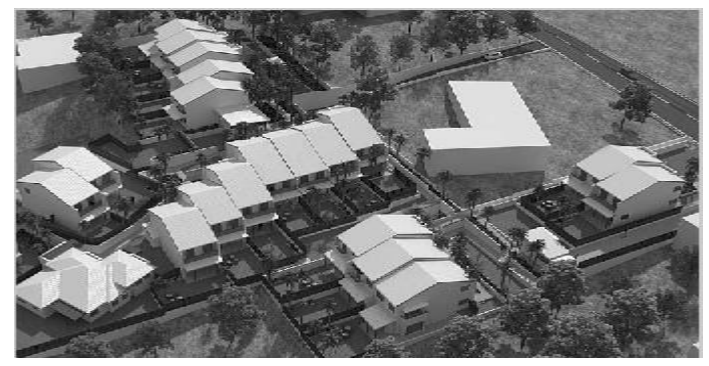

(a)

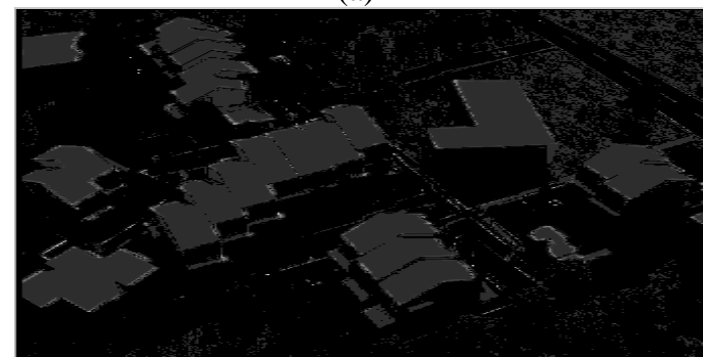

(b)

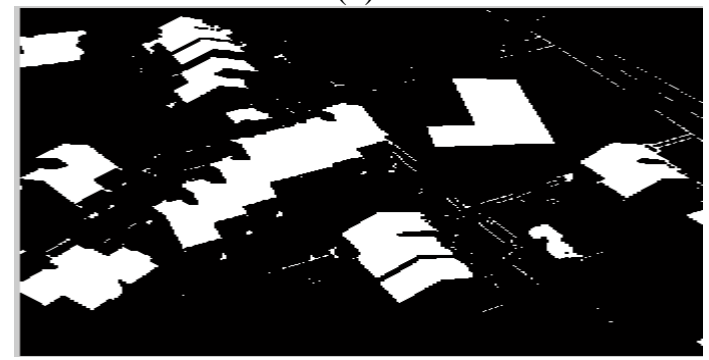

(c)

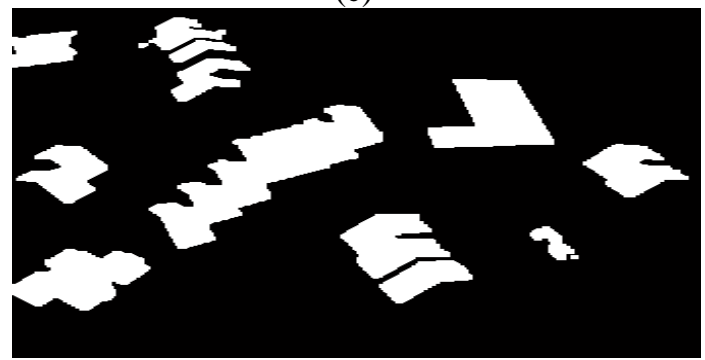

(d)

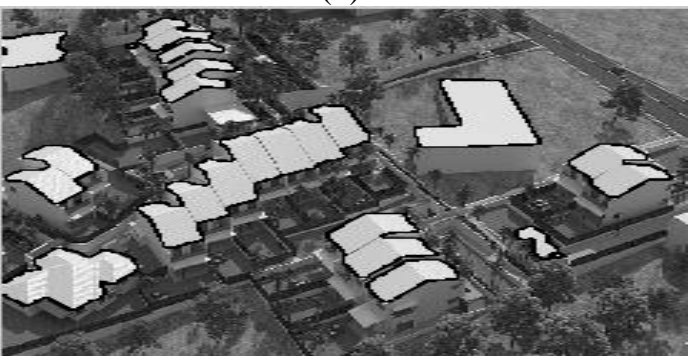

(e)

Figure. 16 Building detection using proposed method: (a) Input building image Building2, (b) Proposed CPMID descriptor result, (c) MOI-FCM based building detection, (d) Post processing result, and (e) Final building detection 
Table 1. MSE analysis for DB-ASTER-GED database

\begin{tabular}{|l|l|l|l|l|}
\hline \multirow{2}{*}{ Images } & \multicolumn{4}{|l|}{ Mean Square Analysis } \\
\cline { 2 - 5 } & BD-RD & BD-MO & BD-HL & Proposed \\
\hline Building1 & 0.160 & 0.119 & 0.097 & $\mathbf{0 . 0 2 2}$ \\
\hline Building2 & 0.164 & 0.104 & 0.074 & $\mathbf{0 . 0 1 8}$ \\
\hline Building3 & 0.132 & 0.093 & 0.065 & $\mathbf{0 . 0 1 5}$ \\
\hline Building4 & 0.092 & 0.058 & 0.050 & $\mathbf{0 . 0 0 9}$ \\
\hline Building5 & 0.088 & 0.063 & 0.048 & $\mathbf{0 . 0 1 4}$ \\
\hline
\end{tabular}

BD-RD $\square$ BD-MO $\square$ BD-HL $\square$ Proposed

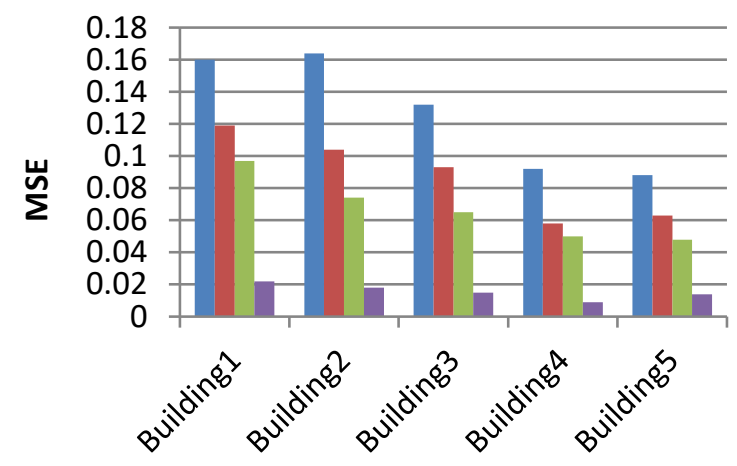

Figure. 17 MSE analysis for DB-ASTER-GED database

Table 2. PSNR analysis for DB-ASTER-GED database

\begin{tabular}{|l|l|l|l|l|}
\hline \multirow{2}{*}{ Images } & \multicolumn{4}{|l|}{ PSNR Analysis(dB) } \\
\cline { 2 - 5 } & BD-RD & BD-MO & BD-HL & Proposed \\
\hline Building1 & 56.089 & 57.375 & 58.263 & $\mathbf{6 4 . 5 3 2}$ \\
\hline Building2 & 55.982 & 57.960 & 59.420 & $\mathbf{6 5 . 4 8 2}$ \\
\hline Building3 & 56.925 & 58.413 & 59.975 & $\mathbf{6 6 . 3 6 9}$ \\
\hline Building4 & 58.460 & 60.481 & 61.132 & $\mathbf{6 8 . 2 1}$ \\
\hline Building5 & 58.686 & 60.116 & 61.237 & $\mathbf{6 6 . 4 8 7}$ \\
\hline
\end{tabular}

Table 3. Segmentation-accuracy analysis for DB-ASTERGED database

\begin{tabular}{|c|c|c|c|c|}
\hline \multirow{2}{*}{ Images } & \multicolumn{4}{|c|}{ Accuracy Analysis (\%) } \\
\cline { 2 - 5 } & BD-RD & BD-MO & BD-HL & Proposed \\
\hline Building1 & 77.91 & 83.77 & 86.21 & $\mathbf{9 2 . 8 7}$ \\
\hline Building2 & 79.74 & 84.90 & 86.92 & $\mathbf{9 3 . 9 4}$ \\
\hline Building3 & 74.13 & 77.89 & 80.71 & $\mathbf{9 0 . 0 1}$ \\
\hline Building4 & 77.99 & 82.21 & 83.63 & $\mathbf{9 1 . 9 2}$ \\
\hline Building5 & 73.19 & 76.88 & 79.95 & $\mathbf{8 8 . 8 3}$ \\
\hline
\end{tabular}

method is BD-HL. Fig.17 represents the MSE outputs of the proposed and existing methods.

The Peak Signal to Noise Ratio analysis is computed from MSE based on Eq. (53). The PSNR analysis is described in Table 2. The high score PSNR refers the best segmentation method and vice versa. The proposed method reaches high PSNR than existing methods. The highest PSNR value acquired by the proposed method is 68.21 . Similar to MSE, the BD-HL method takes the second best position based on PSNR. The PSNR computation is conducted using Eq. (53).

$$
\operatorname{PSNR}\left(I, I^{\prime}\right)=10 \log _{10}\left[\frac{255^{2}}{M S E\left(I, I^{\prime}\right)}\right]
$$

Table 3 and Fig.19 expresses the SegmentationAccuracy analysis which is computed using the segmentation-output and Ground-truth-image. The Segmentation-Accuracy is computed using Eq. (54).

$$
S A=\frac{(T P+T N)}{T P+F P+T N+F N} \times 100
$$

Where
SA-Segmentation Accuracy
TP-True Positive
FP-False Positive
TN-True Negative
FN-False Negative

Here, TP refers the count of pixels that is truly segmented as positive; TN refers to the count of pixels that are truly segmented as negative; FP points out the pixel count that is falsely segmented as positive and FN indicates the pixel count that is falsely segmented as negative. The Segmentation accuracy is computed in percentage. The high score in SA means best segmentation method and vice versa. The efficiency of proposed method is proved by this analysis because of its high segmentation accuracy than existing methods. Here also BD-HL method is second best method based on SA analysis.

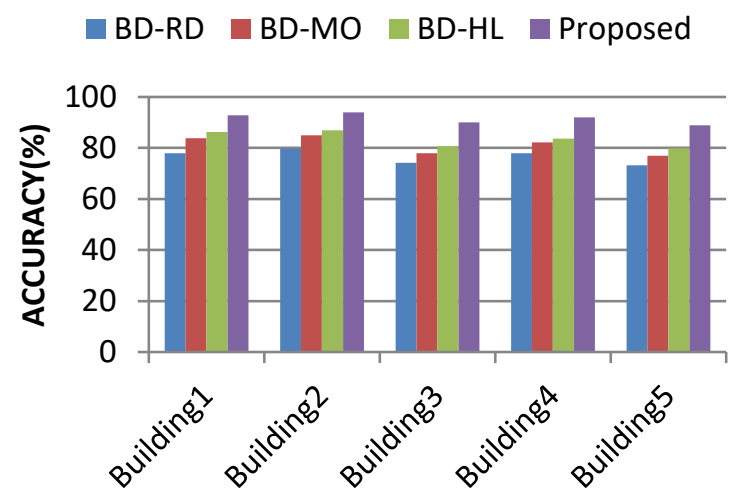

Figure. 19 Segmentation-Accuracy analysis chart

Table 4. Time-taken analysis for DB-ASTER-GED database

\begin{tabular}{|l|l|l|l|l|}
\hline \multirow{2}{*}{ Images } & \multicolumn{4}{|c|}{ Time-taken Analysis(in seconds) } \\
\cline { 2 - 5 } & BD-RD & $\begin{array}{l}\text { BD- } \\
\text { MO }\end{array}$ & BD-HL & Proposed \\
\hline Building1 & 3.91 & 4.97 & 5.17 & $\mathbf{3 . 5 1}$ \\
\hline Building2 & 3.89 & 4.95 & 5.12 & $\mathbf{3 . 4 3}$ \\
\hline Building3 & 3.92 & 5.02 & 5.19 & $\mathbf{3 . 4 9}$ \\
\hline Building4 & 3.81 & 4.96 & 5.10 & $\mathbf{3 . 4 0}$ \\
\hline Building5 & 3.85 & 5.05 & 5.16 & $\mathbf{3 . 4 5}$ \\
\hline
\end{tabular}




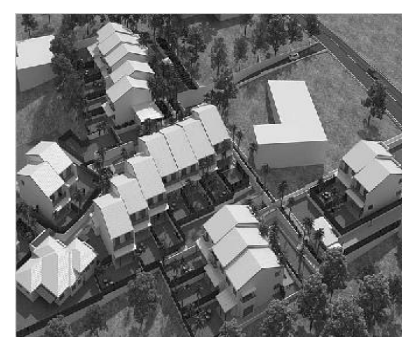

(a)

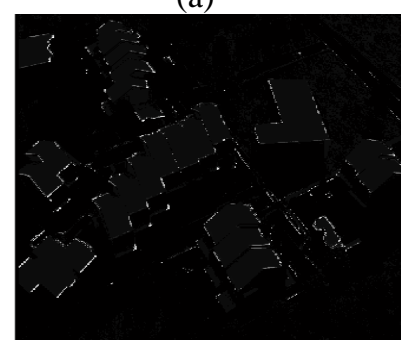

(e)

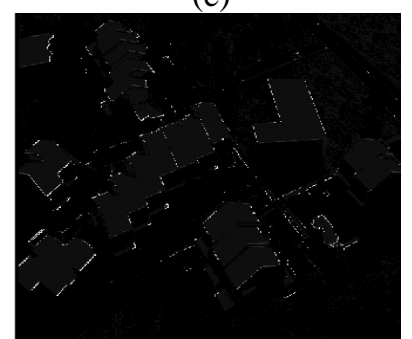

(i)

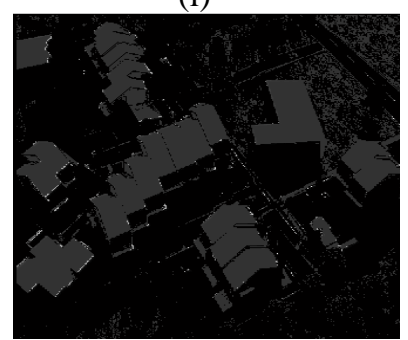

(m)

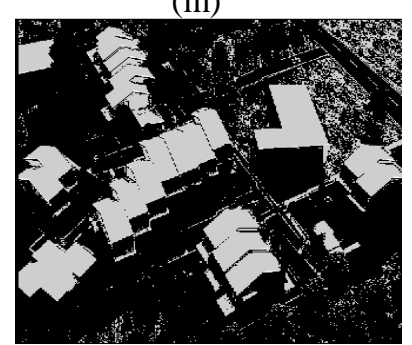

(q)

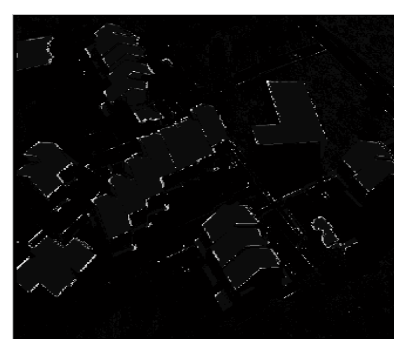

(b)

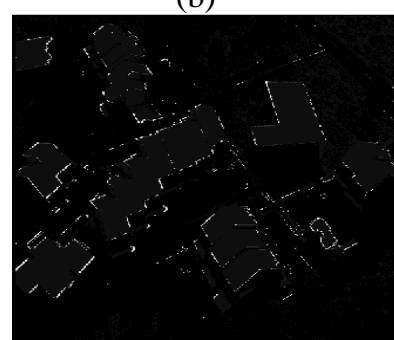

(f)

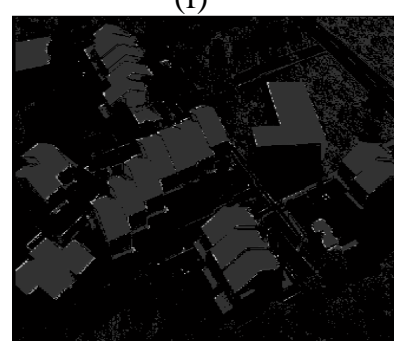

(j)

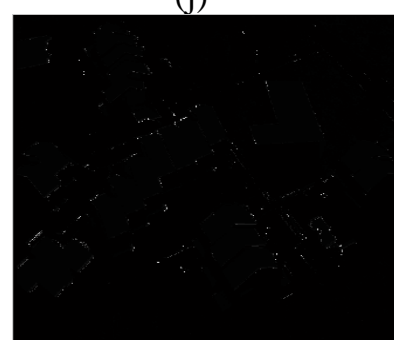

(n)

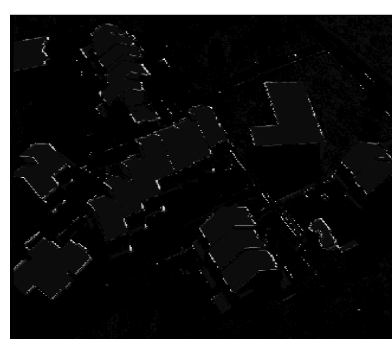

(c)

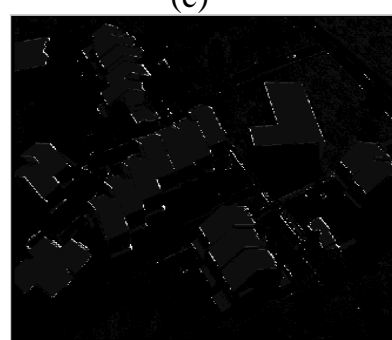

(g)

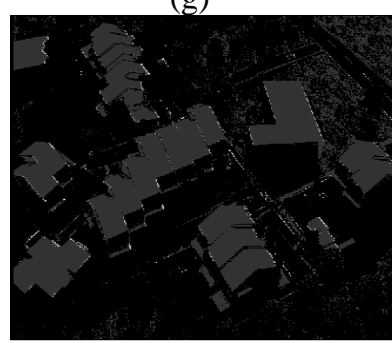

(k)

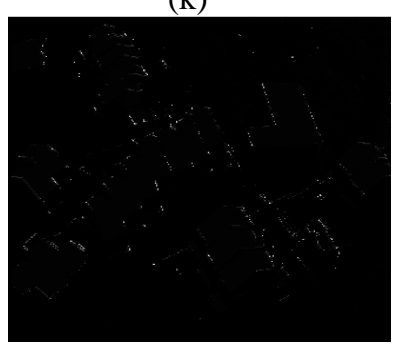

(o)

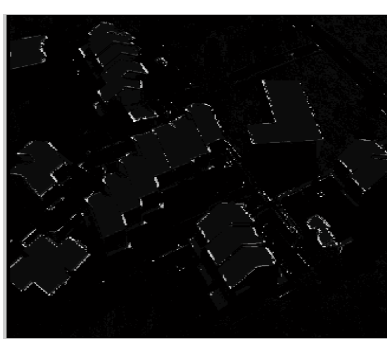

(d)

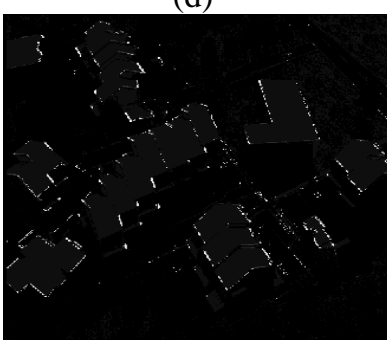

(h)

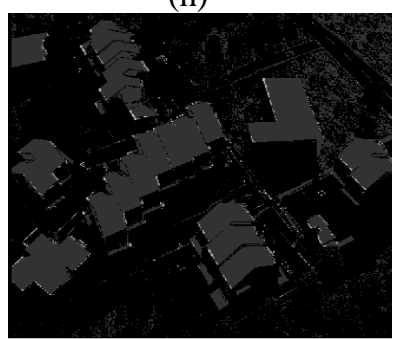

(1)

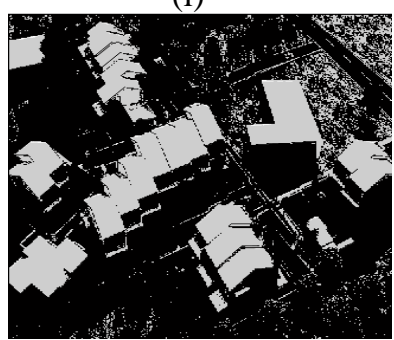

(p)

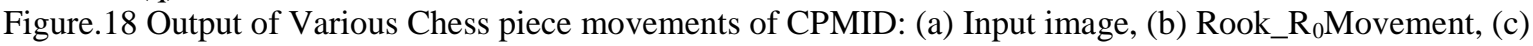
Rook_R ${ }_{1}$ Movement, (d) Rook_R 2 Movement, (e) Rook_R ${ }_{3}$ Movement, (f) Knight_N 0 Movement, (g) Knight_N 1 Movement, (h) Knight_N 2 Movement, (i) Knight_N 3 Movement, (j) Bishop_B 0 Movement, (k) Bishop_B 1 Movement, (l) Bishop_B 2 Movement, (m)Bishop_B 3 Movement, (n) Queen_Q ${ }_{0}$ Movement, (o) Queen_Q ${ }_{1}$ Movement, (p) King_K $\mathrm{K}_{0}$ Movement, and (q) King_K $\mathrm{K}_{1}$ Movement

Table 4 shows the Time taken analysis of the proposed method. From the Table, the proposed method requires less time than the existing methods proving its efficiency in terms of time.

The Table 5 denotes the analysis on Avg. MSE, Avg. PSNR, Avg. Accuracy, Avg. Time taken, Avg.
Specificity and Avg. FScore. The average FScore of proposed method is 0.902 but only 0.868 is the achievement of BD-HL method. The Specificity is computed based on Eq. (55). The FScore measure is computed based on Eqs. (56) and (57). 


$$
\text { Specificity }=\frac{T P}{(T P+F N)}
$$

$$
\text { FScore }=2 \times\left(\frac{\text { Sensitivity } \times \text { Specificity }}{\text { Sensitivity }+ \text { Specificity }}\right)
$$

$$
\text { Sensitivity }=\frac{T P}{(T P+F P)}
$$

Table 5. Performance analysis for DB-ASTER-GED database

\begin{tabular}{|l|l|l|l|l|}
\hline \multirow{2}{*}{ Images } & \multicolumn{4}{|l|}{ Segmentation methods } \\
\cline { 2 - 5 } & $\begin{array}{l}\text { BD- } \\
\text { RD }\end{array}$ & BD-MO & $\begin{array}{l}\text { BD- } \\
\text { HL }\end{array}$ & Proposed \\
\hline $\begin{array}{l}\text { Average } \\
\text { MSE }\end{array}$ & 0.5656 & 0.087 & 0.066 & $\mathbf{0 . 0 1 5 6}$ \\
\hline $\begin{array}{l}\text { Average } \\
\text { PSNR } \\
\text { in dB) }\end{array}$ & 57.228 & 58.869 & 60.005 & $\mathbf{6 6 . 2 1 6}$ \\
\hline $\begin{array}{l}\text { Average } \\
\text { Accuracy } \\
\text { (in \%) }\end{array}$ & 76.592 & 81.13 & 83.484 & $\mathbf{9 1 . 5 1 4}$ \\
\hline $\begin{array}{l}\text { Average } \\
\text { Time } \\
\text { taken(in } \\
\text { seconds) }\end{array}$ & 3.87 & 4.99 & 5.148 & $\mathbf{3 . 4 5 6}$ \\
\hline $\begin{array}{l}\text { Average } \\
\text { Specificity }\end{array}$ & 0.857 & 0.882 & 0.921 & $\mathbf{0 . 9 5 0}$ \\
\hline $\begin{array}{l}\text { Average } \\
\text { FScore }\end{array}$ & 0.8217 & 0.855 & 0.868 & $\mathbf{0 . 9 0 2}$ \\
\hline
\end{tabular}

Table 6. Eye perception quality index (EPQI) analysis for DB-ASTER-GED database

\begin{tabular}{|c|c|c|c|c|}
\hline \multirow{2}{*}{ Images } & \multicolumn{4}{|c|}{ EPQI Analysis } \\
\cline { 2 - 5 } & BD-RD & $\begin{array}{c}\text { BD- } \\
\text { MO }\end{array}$ & BD-HL & Proposed \\
\hline Building1 & 1 & 2 & 3 & $\mathbf{4}$ \\
\hline Building2 & 2 & 1 & 3 & $\mathbf{4}$ \\
\hline Building3 & 1 & 2 & 3 & $\mathbf{4}$ \\
\hline Building4 & 2 & 1 & 3 & $\mathbf{4}$ \\
\hline Building5 & 1 & 2 & 3 & $\mathbf{4}$ \\
\hline
\end{tabular}

The Table 6 shows the analysis of Eye Perception Quality Index. According to this table and table, the proposed method has higher accuracy even better than BD-HL method.

\section{Conclusion}

The proposed BD-CPMIDFM method is a successful solution for the issue of deficiency in accuracy on detection of buildings in urban area. A new descriptor CPMID which has an energized mechanism that uses Chess piece movement directions that can be used as a feature set for the FCM segmentation. The potential of the image descriptor CPMID derives the energetic feature set for Fuzzy classifier. The proposed method is analyzed by the measurement parameters MSE, PSNR, Accuracy and Time-taken. The proposed BD-CPMIDFM is highlighted with least MSE, highest PSNR, highest Accuracy and reasonable time taken. The Highest Accuracy attained by the proposed method is 93.94. The proposed method outperforms the existing methods through better achievements in MSE, PSNR and Accuracy. The shape based features can be additionally integrated with this method to reach extreme performance in building detection.

\section{References}

[1] H. Shi, L. Chen, F. Bi, H. Chen, and Y. Yu, "Accurate Urban Area Detection in Remote Sensing Images", IEEE Geoscience and Remote Sensing Letters, Vol. 12, No. 9, pp. 1948-1952, 2015.

[2] C. Tao, Y. Tan, Z. Zou, and J.W. Tian, "Unsupervised Detection of Built-up areas from multiple high-resolution remote sensing images", IEEE Geosciences and Remote Sensing Letters, Vol. 10, No. 6, pp. 1300-1304, 2013.

[3] B. Sirmacek and C. Unsalan, "Urban area and building using SIFT key points and graph theory", IEEE Transactions on Geosciences and Remote Sensing, Vol. 47, No. 4, pp. 11561167, 2009.

[4] S. Chattopadhyay, D. K. Prathihar, and S.C. DeSarkar, "A comparative study of fuzzy CMeans algorithm and entropy-based fuzzy clustering algorithms", Computing and Informatics, Vol. 30, pp. 701-720, 2011.

[5] S. Arora and R. Pandey, "Applications of morphological operators using image morphological algorithms", SSRG International Journal of Electronics and Communication Engineering, Vol. 3, No. 8, pp. 107-110, 2016.

[6] S. Chen, H. Wang, F, Xu, and Y.Q. Jin, "Automatic recognition of isolated buildings on single Aspect SAR image using range detector", IEEE Geosciene and Remote Sensing Letters, Vol. 12, No. 2, pp. 219-223, 2015.

[7] Q. Zhang, X. Huang, and G. Zhand, "A morphological building detection framework for high-resolution optical imagery over urban areas", IEEE Geosciene and Remote Sensing Letters, Vol. 13, No. 9, pp. 1388-1392, 2016.

[8] D. Konstantinidis, T. Stathaki, V. Argyriou, and N. Gvammalidis, "Building detection using enhanced HOG-LBP features and region refinement process", IEEE Journal of Selected Topics in Applied Earth Observations and 
Remote Sensing, Vol. 10, No. 3, pp. 888-905, 2017.

[9] ASTER_GED Dataset Website https://lpdaac. usgs.gov/about/news_archive/aster_global_emi ssivity_dataset_ged_product_release, 2017.

[10] J. D. Wegner, R. Hansch, A. Thiele, and U. Soergel, "Building detection from One orthophoto and high-resolution InSAR data using conditional random fields", IEEE Journal of Selected Topics in Applied Earth Observations and Remote Sensing, Vol.4, No. 1, pp. 83-91, 2011.

[11] [11] A. Turlapaty, B. Gokaraju, Q. Du, N. H.Youman and J. V. Aanstoos, "A hybrid approach for building extraction from space borne multi-angular optical imagery", IEEE Journal of selected topics in applied earth observations and remote sensing,vol.5, No. 1, pp. 89-100,2012.

[12] A. Ferro, D. Brunner, and L. Bruzzone, "Automatic detection and reconstruction of building radar footprints from single VHR SAR images", IEEE Transactions on Geoscience and Remote Sensing, Vol. 51, No. 2, pp. 935952, 2013.

[13] C. Tao, Y. Tan, Z. Zou, and J. Tian, "Unsupervised detection of built-up areas from multiple high-resolution remote sensing images", IEEE Geoscience and Remote Sensing Letters, Vol. 10, No. 6, 2013.

[14] L. Deng and C. Wang, "Improved building extraction with integrated decomposition of time-frequency and entropy- alpha using polarimetric SAR data", IEEE Journal of Selected Topics in Applied Earth Observations and Remote Sensing, Vol. 7, No.10, pp. 40584068, 2014

[15] H.C. Fan, W. Yao, and L. Tang, "Identifying man-made objects along urban road corridors from mobile LiDAR data", IEEE Geoscience and Remote Sensing Letters, Vol. 11, No. 5, pp. 950-954, 2014.

[16] W. Wu, H. Guo, and X. Li, "Urban area manmade target detection for PolSAR data based on a nonzero-mean statistical model", IEEE Geoscience and Remote Sensing Letters, Vol.11, No. 10, pp. 1782-1786, 2014.

[17] N. Li, F. Liu, and Z. Chen, "A texture measure defined over intuitionistic fuzzy set theory for the detection of built-up areas in high resolution SAR images", IEEE Journal of Selected Topics in Applied Earth Observations and Remote Sensing, Vol.7, No. 10, pp. 4255-4265, 2014.

[18] W.J. Wu, H. Guo, and X. Li, "Urban area SAR image man-made target extraction based on the product model and the time-frequency analysis", IEEE Journal of Selected Topics in Applied Earth Observations and Remote Sensing, Vol. 8, No. 3, pp. 943-952, 2015.

[19] J. Gu, L. Jiao, S. Yang, and F. Liu, "Fuzzy double C-means clustering based on spare selfrepresentation", IEEE Transactions on Fuzzy Systems, Vol. P, No. 99, 2017.

[20] F. Li and J. Qin, "Robust Fuzzy local Information and Lp-norm distance based image segmentation method", IET Image Processing, Vol. 11, No. 4, pp. 217-226, 2017.

[21] K. H. Memon and D. H. Lee, "Generalised fuzzy C-means clustering algorithm with local information", IET Image Processing, Vol. 11, No.1, pp. 1-12, 2017.

[22] H. Zhang, Q. Wang, W. Zhangshi, and M. Hao, "A novel adaptive fuzzy local information Cmeans clustering algorithm for remotely sensed imagery classification", IEEE Transactions on Geoscience and Remote Sensing, Vol. 55, No. 9, pp. 5057-5068, 2017.

[23] J.C. Bezdek and J.C. Dunn, "Optimal fuzzy partitions: a huristic for estimating the parameters in a mixture of normal distributions", IEEE Transactions on Computer, Vol. C-24, No. 8, pp.835-838, 1975.

[24] J.C. Bezdek, "Pattern recognition with fuzzy objective function algorithms", Plenum Press Newyork,1981.

[25] Morphological operators: www0.cs.ucl.ac.uk/staff/a.13rostow/classes/IP 2008/L3-Morphology.pdf

[26] Morphological Operators: www. Coe.utah.edu/cs4 640/slides/lecture11.pdf

[27] S. Arora and R. Pandey, "Applications of morphological operators using image morphological algorithms", SSRG international Journal of Electronics and Communication Engineering, Vol.3, No.8, pp. 107-110, 2016.

[28] D. E. Ilea, P. F. Whelan, and O. Ghita, "Performance characterization of clustering algorithms for color image segmentations", In: Proc. of the $10^{\text {th }}$ International Conference on Optimization of Electrical and Electronic Equipment, pp.18 - 19, 2006. 